\title{
Effects of fatiguing isometric and isokinetic ankle exercises on postural control while standing on firm and compliant surfaces
}

\author{
Etienne J Bisson ${ }^{1,2^{*}}$, Anthony Remaud ${ }^{2 \dagger}$, Sébastien Boyas ${ }^{1,2 \dagger}$, Yves Lajoie $^{1 \dagger}$ and Martin Bilodeau ${ }^{1,2,3 \dagger}$
}

\begin{abstract}
Background: Fatiguing exercises used across studies to induce alterations in postural control are diverse and may explain the different findings reported. This study aimed to compare the effects of two types of fatiguing plantarflexion exercises on postural control on a firm and a compliant surface. Ten healthy young men ( $29 \pm 4$ years) were asked to stand as steadily as possible for $30 \mathrm{~s}$, blindfolded with feet together, on a firm and a compliant surface before and immediately after an isometric and an isokinetic fatiguing exercise.

Results: Maximal force reduction due to fatigue was found significant but similar between exercises. No significant difference was found between the fatiguing exercises on all Center of Pressure (CoP) parameters. Both fatiguing exercises induced increases in CoP excursion area, CoP variability and CoP velocity in both planes (antero-posterior, mediolateral) on the compliant surface. On the firm surface, both fatiguing exercises only induced increases in CoP variability and CoP velocity in the fatigued plane (antero-posterior).

Conclusions: Isometric and isokinetic fatiguing exercises, when producing a similar level of force reduction, induce similar decreases in postural control. The effects of fatigue on postural control in healthy young men are more pronounced when standing on a compliant surface, i.e. when proprioceptive information at the ankle is altered.
\end{abstract}

Keywords: Neuromuscular fatigue, Posturography, Centre of Pressure, Contraction mode, Plantarflexor muscles

\section{Background}

The ability to perform a multitude of physical activities and most daily living activities requires adequate postural control. In order to maintain postural control, the central nervous system must integrate and (re-)weigh information from the visual, vestibular and somatosensory systems and modulate commands to the neuromuscular system continuously [1]. Not surprisingly, numerous studies [2-11] have demonstrated an impairment in postural control in different postural tasks after neuromuscular fatigue. Since the plantarflexor muscles constitute the main controllers in quiet standing [12], the effect of fatigue of these muscles on postural control

\footnotetext{
* Correspondence: ebisson@uottawa.ca

${ }^{\dagger}$ Equal contributors

'School of Human Kinetics, Faculty of Health Sciences, University of Ottawa,

Ottawa, Canada

${ }^{2}$ Aging and Movement Research Laboratory, Bruyère Research Institute,

Ottawa, Canada

Full list of author information is available at the end of the article
}

have been largely examined. The findings, however, vary significantly possibly due to methodological differences (i.e. fatigue protocols, postural stances used and/or amount of visual information available) across studies. In a recent study [4], we found that an isometric fatiguing exercise (standing on tip-toes until exhaustion) increased Center of Pressure (CoP) excursion area, CoP variability and $\mathrm{CoP}$ velocity in both antero-posterior (AP) and medio-lateral (ML) planes. This increase was found independent of the difficulty of the postural task (unipedal, tandem, bipedal), except for a more pronounced effect for the most challenging postural task (i.e. unipedal stance with eyes closed). Our results were consistent with other studies using a similar isometric fatiguing exercise [5,8-11]. In contrast, when the ankle musculature was fatigued with an isokinetic exercise in a different study [2], an increase in CoP velocity was found only in the AP plane, i.e. the plane in which the fatigued plantarflexor muscles act as prime mover muscles. Again, these results were consistent with studies that have used a similar fatiguing

\section{Biomed Central}


exercise $[3,6,13]$. In addition to the different modes of contraction (isometric vs. isokinetic), other parameters specific to a given fatiguing exercise (intensity, duration) can explain why results vary across studies. Thus, it can be questioned if the impairment of postural control is really influenced by the mode of contraction or if it is only a factor of intensity and/or duration of the fatigue task.

Intense or prolonged exercise inevitably induces neuromuscular fatigue through various mechanisms, i.e., alterations in the activation of the primary motor cortex, reduction of motor unit discharge rates, alterations of excitation-contraction coupling, and slowing of the contractile apparatus [14,15]. Furthermore, muscle fatigue due to intense or prolonged exercise also affects the proprioceptive system [16,17]. This could be explained by the accumulation of metabolites leading to altered muscle spindle function [17], as well as altered central processing of proprioception via group III and IV afferents [16]. However, it has been shown that the mechanisms involved in muscle fatigue are dependent on the exercise performed to fatigue the muscles (task dependency) [18]. Sustained isometric activities have been suggested to induce greater central fatigue compared to concentric intermittent contractions due to greater metabolite by-products accumulation with the limited blood flow, causing in turn an inhibition of the supraspinal descending drive possibly via small-diameter afferents $[14,15,19]$. However, the impact of these different modes of contraction on postural control has yet to be compared.

Compliant surfaces (such as temper foams and swayreferenced platforms) are commonly used in postural studies to examine the reliance on proprioceptive information in the control of postural tasks [1,20-25]. They are also used in clinical settings to assess balance [26]. By comparing CoP parameters on a firm and a compliant surface, the latter condition has been shown to be more sensitive to balance problems observed with old age $[22,25]$, pathologies such as vestibular disorder [1] and injuries [20,21]. Since a reduction in proprioception and the re-weighting of this information have been proposed to be the main causes of decreased postural control with fatigue [27], one may expect the effect of fatigue to be different on a firm versus a compliant surface. Several studies [7,28-30] have shown an impairment in postural control when standing on a compliant surface (sway-referenced platform) after fatigue. However, only one study [30] compared fatigue-related changes of CoP parameters between a compliant and a firm surface. The authors found an impairment in postural control after ankle, knee and global fatigue. This effect was not greater on a sway-referenced platform. Since the results of this study may be limited because of methodological issues (i.e. duration and number of trials), more research is needed to clarify these findings.
Thus, in an attempt to elucidate factors contributing to the different reports regarding the effect of fatigue on postural control, the objectives of the present study were: a) to compare the effects of isometric and concentric isokinetic fatiguing exercises on postural control during quiet stance, and b) to assess whether the magnitude of these effects are dependent on the amount of proprioceptive information available (i.e., by comparing postural tasks performed on a firm surface and a compliant surface). It was hypothesized that an isometric fatiguing exercise would induce greater alterations in postural control compared to an isokinetic concentric fatiguing exercise, likely due to a greater increase in metabolites concentration [19]. It was further hypothesized that postural control on a compliant surface would be impaired to a greater extent after fatigue compared to a firm surface because standing on a compliant surface requires more proprioceptive information and greater control by the neuromuscular system.

\section{Results \\ Type of fatigue \\ $M V C_{I M}$}

As shown in Figure 1, results on the $\mathrm{MVC}_{\mathrm{IM}}$ showed a significant main effect of fatigue $\left(\mathrm{F}=94.6, \mathrm{p}<0.001, \eta_{\mathrm{p}}^{2}=\right.$ $0.91)$. The main effect of type of fatigue $\left(F=2.6, p=0.14, \eta_{p}^{2}\right.$ $=0.22)$ and the fatigue by type interaction $(\mathrm{F}=1.3, \mathrm{p}=0.30$, $\left.\eta_{\mathrm{p}}^{2}=0.12\right)$ were found not significant. $\mathrm{MVC}_{\mathrm{IM}}$ recorded after each fatiguing exercises (Pooled IM and IK mean values for post-fatigue $1=180.3 \pm 34.5 \mathrm{~N} \cdot \mathrm{m}$ and for post-fatigue $2=$ $175.8 \pm 32.9 \mathrm{~N} \cdot \mathrm{m})$ were significantly different from prefatigue (Pooled IM and IK mean values $=246.9 \pm 42.0 \mathrm{~N} \cdot \mathrm{m}$; $\mathrm{p}<0.001)$. The absence of the effect of the type of fatigue suggests that the level of fatigue was similar between the IM

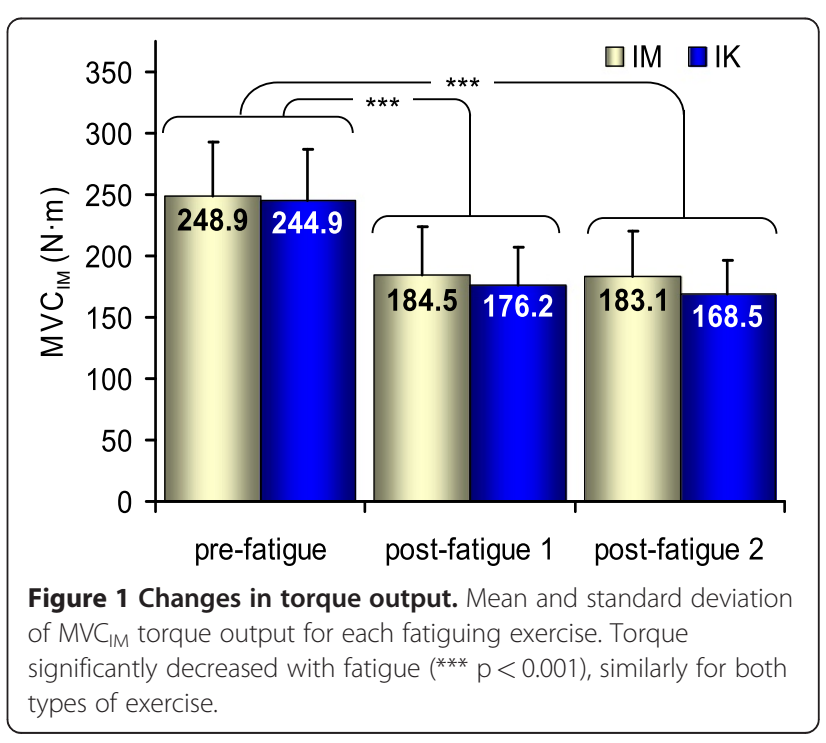


and $\mathrm{IK}$ fatiguing exercises (mean \% of pre-fatigue $\mathrm{MVC}_{\mathrm{IM}}=$ $73.9 \pm 6.7$ and $70.9 \pm 8.7$, respectively).

\section{Time to failure}

Results showed significant main effects of fatigue ( $\mathrm{F}=$ $\left.11.4, \mathrm{p}=0.01, \eta_{\mathrm{p}}^{2}=0.59\right)$, type of fatigue $(\mathrm{F}=39.5$, $\mathrm{p}=0.001, \eta_{\mathrm{p}}^{2}=0.83$ ), and a type by fatigue interaction $\left(\mathrm{F}=17.1, \mathrm{p}=0.003, \eta_{\mathrm{p}}^{2}=0.68\right)$. The time to failure of the plantarflexor muscles was significantly longer during the IM session than during the IK session for the first bout of fatiguing exercise (respectively $145.3 \pm 34.6$ s vs. $54.6 \pm 12.1 \mathrm{~s}, \mathrm{p}<0.001$ ) and for the second bout of fatiguing exercise (respectively $107.0 \pm 39.9$ s vs. $56.2 \pm$ $13.6 \mathrm{~s}, \mathrm{p}=0.002$ ). In addition, the time to failure of the first bout of fatiguing exercise was longer compared to the second bout of fatiguing exercise for the IM mode $(\mathrm{p}=0.003)$, but not for the IK mode $(\mathrm{p}=0.57)$.

\section{Effect of fatigue on CoP parameters}

For all CoP parameters analyzed, the effect of the type of fatigue (IM vs. IK) and all relevant interactions were found non-significant ( $p>0.05)$. Thus, data from both type of fatiguing exercises were pooled and the subsequent results only describe the effects of fatigue (pre and post-fatigue) and surface (firm, compliant) on the CoP parameters.

\section{CoP excursion area}

Main effects of fatigue $\left(F=7.9, \mathrm{p}=0.02, \eta_{\mathrm{p}}^{2}=0.47\right)$, and surface $\left(\mathrm{F}=91.8, \mathrm{p}<0.001, \eta_{\mathrm{p}}^{2}=0.91\right)$ were found significant for the $95 \%$ ellipse area parameter. A significant fatigue by surface interaction $\left(\mathrm{F}=12.6, \mathrm{p}=0.006, \eta_{\mathrm{p}}^{2}=0.58\right)$ was also found. As shown in Figure 2, CoP excursion area was significantly greater during the compliant condition

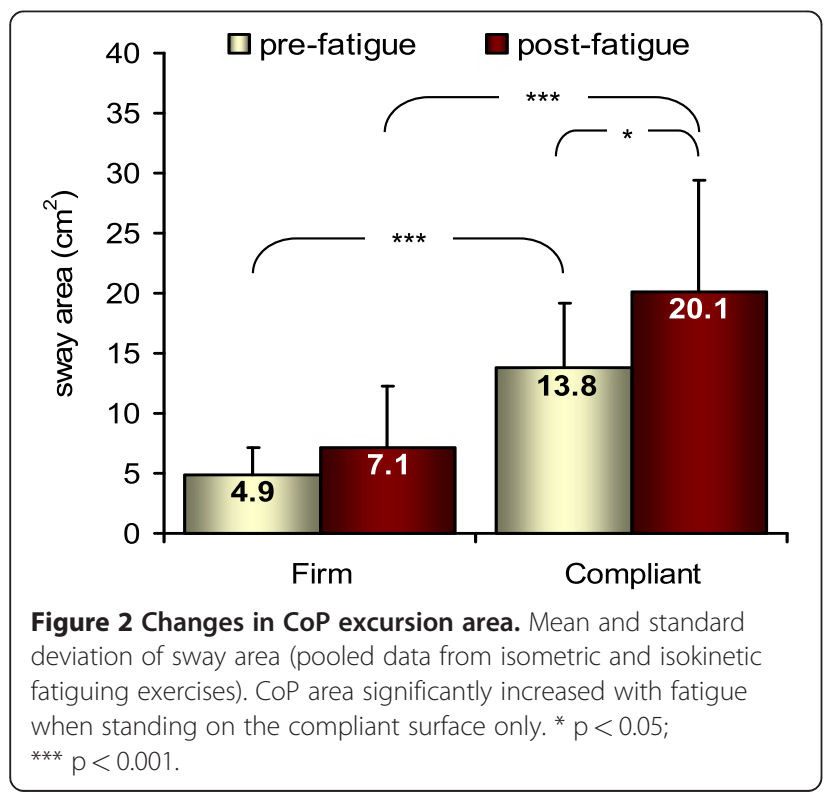

compared to the firm condition in both pre and postfatigue $(\mathrm{p}<0.001)$. Also, the effect of fatigue depended on the difficulty of the task (firm vs. compliant surface) since $\mathrm{CoP}$ excursion area increased significantly after fatigue when standing on the compliant surface $(\mathrm{p}=0.011)$ but not on the firm surface $(\mathrm{p}=0.108)$.

\section{$M L$ postural control}

Figure 3 shows the results for $\mathrm{ML} \mathrm{CoP}$ velocity (Figure $3 \mathrm{~A}$ ) and variability (Figure 3B). A main effect of fatigue $\left(F=12.41, p=0.006, \eta_{p}^{2}=0.58\right)$ was found significant for ML CoP velocity only. Both CoP velocity and $\mathrm{CoP}$ variability showed a significant main effect of surface (velocity: $\mathrm{F}=154.9, \mathrm{p}<0.001, \eta_{\mathrm{p}}^{2}=0.95$; variability: $\mathrm{F}=$ $\left.379.9, \mathrm{p}<0.001, \eta_{\mathrm{p}}^{2}=0.98\right)$ and a fatigue by surface interaction (velocity: $\mathrm{F}=19.9, \mathrm{p}=0.002, \eta_{\mathrm{p}}^{2}=0.69$; variability: $\left.\mathrm{F}=12.7, \mathrm{p}=0.006, \eta_{\mathrm{p}}^{2}=0.59\right)$. As shown in Figure 3, ML $\mathrm{CoP}$ velocity and ML CoP variability was significantly

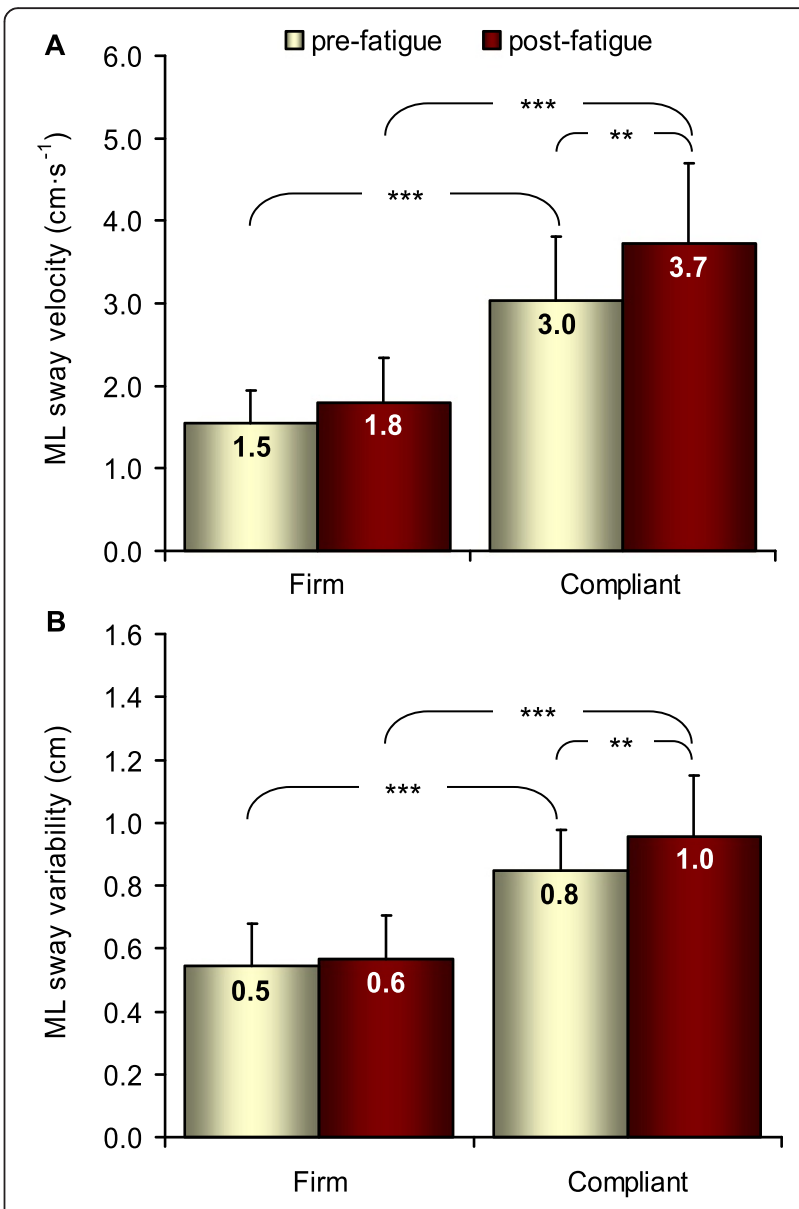

Figure 3 Changes in ML CoP velocity and variability. Mean and standard deviation of CoP parameters in ML (pooled data from isometric and isokinetic fatiguing exercises). ML CoP velocity (A) and ML CoP variability (B) significantly increased with fatigue when standing on the compliant surface only. ${ }^{* *} p<0.01 ;{ }^{* *} p<0.001$. 
greater during the compliant condition compared to the firm condition in both pre and post-fatigue $(\mathrm{p}<0.001)$. Also, the effect of fatigue depended on the difficulty of the task (firm vs. compliant surface) since ML CoP velocity and ML CoP variability increased significantly after fatigue when standing on the compliant surface (ML CoP velocity: $\mathrm{p}=0.003$; $\mathrm{ML}$ CoP variability: $\mathrm{p}=0.003$ ) but not on the firm surface (ML CoP velocity: $\mathrm{p}=0.13$; $\mathrm{ML} \mathrm{CoP}$ variability: $\mathrm{p}=0.90)$.

\section{AP postural control}

Both AP CoP velocity and AP CoP variability showed significant main effects of fatigue (velocity: $\mathrm{F}=13.5, \mathrm{p}=0.005$, $\eta_{\mathrm{p}}^{2}=0.60$; variability: $\left.\mathrm{F}=9.9, \mathrm{p}=0.012, \eta_{\mathrm{p}}^{2}=0.52\right)$ and surface (velocity: $F=125.8, \mathrm{p}<0.001, \eta_{\mathrm{p}}^{2}=0.93$; variability: $\left.\mathrm{F}=193.3, \mathrm{p}<0.001, \eta_{\mathrm{p}}^{2}=0.96\right)$. A significant fatigue by surface interaction was found $\left(\mathrm{F}=7.51, \mathrm{p}=0.023, \eta_{\mathrm{p}}^{2}=\right.$ $0.46)$ for CoP velocity only. AP CoP variability was significantly greater during the compliant condition $(0.91 \pm 0.07$ $\mathrm{cm})$ compared to the firm condition $(0.53 \pm 0.05 \mathrm{~cm})$ and after fatigue $(0.82 \pm 0.08 \mathrm{~cm})$ compared to before fatigue $(0.67 \pm 0.05 \mathrm{~cm})$. As shown in Figure 4, AP CoP velocity was significantly greater during the compliant condition compared to the firm condition in both pre and postfatigue ( $\mathrm{p}<0.001)$. Also, AP CoP velocity increased significantly after fatigue when standing on the compliant surface $(\mathrm{p}=0.007)$ and on the firm surface $(\mathrm{p}=0.005)$. Thus, the significant interaction suggests that AP CoP velocity was increased to a greater extent after fatigue when standing on the compliant compared to the firm surface.

\section{Discussion}

This study compared the effects of two types of fatiguing exercises on postural control while standing on a firm

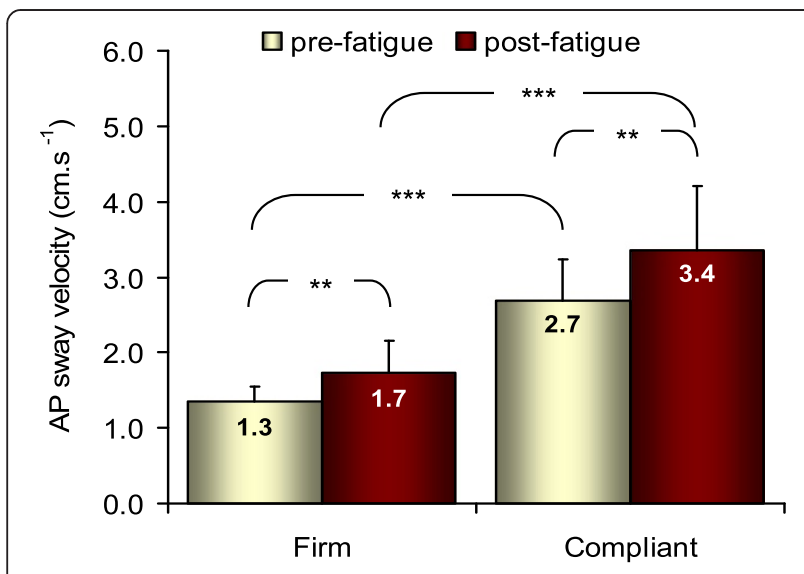

Figure 4 Changes in AP CoP velocity. Mean and standard deviation of AP CoP velocity (pooled data from isometric and isokinetic fatiguing exercises). AP CoP velocity significantly increased with fatigue for both surface conditions but appears to be greater when standing on compliant surface. ${ }^{* *} p<0.01$; ${ }^{* *} p<0.001$. and a compliant surface. Our results showed similar amounts of force reduction and decreased postural control between the two fatiguing exercises performed (isometric vs. isokinetic). This refutes our first hypothesis that an isometric fatiguing exercise would induce greater alterations in postural control compared to an isokinetic concentric fatiguing exercise. As expected, all CoP parameters increased when standing on a compliant surface compared to a firm surface. In addition, all five CoP parameters increased with fatigue when standing on a compliant surface whereas only two parameters (those specific to the fatigued AP plane) increased with fatigue when standing on a firm surface. Furthermore, subjects seem to have a greater increase in AP CoP velocity with fatigue when standing on a compliant compared to a firm surface. This confirms our second hypothesis that postural control on a compliant surface with eyes closed is more impaired after fatigue compared to a firm surface.

\section{Effects of the type of fatigue}

Both contraction types induced the same amount of force reduction but the isometric fatiguing exercise was of longer duration. This difference could have led to a different effect on postural control. However, no difference in postural control changes with fatigue was observed between contraction modes in this study.

Specific characteristics of the fatiguing exercises could explain the absence of a difference concerning their effect on postural control. For example, the combination of intensity (moderate) and overall duration (relatively short) of both types of fatiguing exercises may have been too similar to lead to differences in fatigue mechanisms (central or peripheral [15]) and consequently on postural control. Time to failure has been reported to be faster with higher-intensity exercises [31]. This, combined with the fact that the transition time between the end of the fatiguing exercises and the post-fatigue measurements may have allowed subjects to partly recover from fatigue, could have reduced our ability to detect a difference in the fatigue effects of both contraction modes used.

However, the fact that fatigue did have a significant impact on postural control, suggests that recovery was not complete. Considering that the force of the plantarflexors was reduced to $75 \%$ of the pre-fatigue $\mathrm{MVC}_{\mathrm{IM}}$ and static postural control requires forces of approximately only $10 \%$ MVC [32], it becomes apparent that the reduction in force may not be a major factor explaining the reduction in postural control due to fatigue. Others have suggested that the reduction in postural control due to fatigue may be due to mechanisms other than force-generating capacity, such as altered proprioceptive inputs $[8,9,11]$. Since the intensity of the fatiguing exercises and their effects on postural control were similar, the proprioceptive deficits might have been 
equivalent. Our results also suggest that the seemingly different effect of fatigue observed between isometric and isokinetic activities in the literature might be due to methodological factors. Most isometric fatiguing exercises [4,9-11] have typically been of relatively low intensity (lifting body weight) and have been performed directly on the force platform or fairly close to it, leading to minimal recovery between the end of the fatiguing exercise and the start of postural sway acquisition. In contrast, most studies using isokinetic fatiguing exercises $[2,6,7]$ have used a relatively high intensity $(50 \% \mathrm{MVC})$ and were performed on a dynamometer. Thus, a greater transition time likely occurred and increased the recovery from fatigue prior to the postural testing. Considering the level of intensity (50\%) and the use of a dynamometer to induce fatigue, the results (on firm surface) obtained in the present study reflects those reported in studies that used an isokinetic fatiguing exercises. It would be interesting to examine the differences between modes of contraction using a low force fatiguing exercises.

\section{Effects of altered proprioceptive information}

In a young healthy population, simple postural tasks can be controlled even when some sensory systems are unavailable or altered [1,25,33-36]. According to the sensory reweighting hypothesis $[1,34,36]$, this is possible since all three sensory systems (visual, vestibular and somatosensory) give redundant information and, "the central nervous system dynamically and selectively adjusts the relative contributions of sensory inputs (i.e. the sensory weights) to maintain upright stance depending not only on the sensory environment, but also on the neuromuscular constraints acting on the subject"[35]. However, these studies showed that postural control decreases considerably when the availability of two sensory systems (somatosensory and vision) are reduced during a standing task [1,25,33-36]. Overall, our results are in line with this sensory reweighting hypothesis $[1,34,36]$. Subjects were able to maintain their balance, but all $\mathrm{CoP}$ parameters tested in this study increased when standing blindfolded on a compliant surface.

However, the original finding in this study is that postural control decreased even more after an ankle fatiguing exercise when the availability of these two sensory systems was reduced. Indeed, both fatiguing exercises increased $\mathrm{CoP}$ parameters in both planes (AP and ML) when standing on the compliant surface compared to standing on a firm surface, where CoP parameters increased only in the fatigued plane (AP). In contrast, Dickin and Doan [30] found impairments in postural control due to fatigue (ankle, knee and global fatigue) and type of surface (stable and sway-referenced platform) but no interaction between the two factors. In this latter study, only one trial and one postural parameter (RMS) were used, increasing the variability and potentially decreasing the reliability of the measurements. This may have masked a potential interaction between fatigue and surface type since, according to Ruhe and colleagues [37], at least three trials and parameters in both distance and time-distance domains are needed to accurately characterize postural control.

Vuillerme and colleagues [9] found that vibration of the ankle plantarflexors after fatigue did not show greater effects on postural control compared to fatigue or vibration only. These authors explained their findings by a reduction in sensitivity to the mechanical vibration due to alteration of ankle proprioception (muscle spindles) during fatigue. Since both muscle fatigue and vibration alter the same postural control elements (somatosensory information from the ankle), a combination of the two would not induce greater postural impairments. Conversely, we found in the present study that when subjects were standing on a compliant surface after a fatiguing exercise, their somatosensory information was less reliable potentially due to two distinct mechanisms. First, standing on a compliant surface alters the somatosensory information at the ankle (joint receptors, Golgi tendon organ, muscle spindles) and the sole of the foot (cutaneous mechanoreceptors) in relation to the environment, thus, increasing reliance on other sensory systems (i.e. vestibular) [33]. Second, after fatiguing exercises, the processing of information concerning joint position is altered [38] and force production of the fatigued muscle(s) may be less accurate and more variable [39]. Thus, in the present study, when the kinesthetic references were altered (compliant condition) and muscle position and force sense were impaired (after fatigue), subjects showed greater postural impairments in both planes compared to either condition taken separately. Furthermore, fatiguing the plantarflexor muscles resulted in a decrease in postural control in the ML plane when standing on a compliant but not a firm surface. This is interesting given that postural control in this plane is thought to involve primarly proximal muscles (i.e. hip and back muscles) [40]. However, it has been shown that the contribution of the ankle musculature for quiet standing is still relatively high when feet are less than $8 \mathrm{~cm}$ apart [41]. Although the primary muscle group fatigued was the plantarflexors, some of these muscles can also generate torque in the ML plane. Thus, the role of ankle muscles in maintaining postural control in the ML plane with feet together may have been altered after fatigue. To compensate for this, subjects may have re-weighted their sensory inputs to the hip musculature. This strategy may have been effective on a firm surface but not on a compliant surface where the ankle musculature is more solicited.

Although this study was limited to young healthy adults, the impact of these results could be important if found with a more vulnerable population (i.e. older adults, diabetic 
patients with peripheral neuropathy). These populations, with a potentially less reliable somatosensory system, could be more at risk of falls/injuries following muscle fatigue. Future studies should focus on the impact of muscle fatigue in a population with reduced proprioception.

\section{Conclusions}

Previous studies on the effect of ankle fatigue on postural control have shown changes in CoP parameters of different magnitude using different types of fatiguing exercises (different mode of contractions, duration, intensities). The present study aimed to compare the effect of two types of exercise (isometric, isokinetic) with a similar intensity on postural control. We found that isometric and isokinetic fatiguing exercises performed until a similar force reduction induce a similar decrease in postural control. This finding suggests that the decrease in postural control due to muscle fatigue is independent of the mode of contraction performed. Thus, differences between studies are likely due to other factors, such as differences in the force reduction induced by the fatiguing activity, and not due to the type of contraction used. Furthermore, the effects of fatigue on postural control were more pronounced when standing on a compliant surface, i.e. when proprioceptive information at the ankle was altered. This finding supports the literature suggesting that postural control deficits observed after fatigue are largely due to a deficit in proprioception, and suggests that fatigued-related balance impairments leading to falls/injuries could be more frequent in situations where proprioceptive information is reduced (e.g. diabetic or other patient with peripheral neuropathy).

\section{Methods}

\section{Subjects}

Ten healthy young men $(29 \pm 4$ years, $178.6 \pm 7.5 \mathrm{~cm}$, $73.6 \pm 9.6 \mathrm{~kg}$ ) were recruited to participate in this study. Subjects had no neurological problems and no history of falls or ankle injury in the past year. The study was approved by the University of Ottawa and the Bruyère Continuing Care research ethics boards, and written informed consent was obtained from each subject prior to their participation in the study.

\section{Procedures}

Subjects took part in two identical sessions, performed one week apart, where postural control was assessed before and immediately after an isometric (IM) or isokinetic (IK) plantarflexion fatiguing exercise. For both sessions (IM session and IK session), postural control was assessed under two surface conditions (firm versus compliant surface), where three 30-s trials of standing as still as possible with feet together and blindfolded (opaque ski goggles) were collected before and after fatigue. This postural task (feet together) without vision was shown to be more sensitive to fatigue on a firm surface compared to the same task with vision $[4,8]$. Thus, 3 postural trials $\times 2$ types of surface (firm and compliant) $\times 2$ time points (before and after fatigue) for a total of 12 trials were performed per session. A medium density temper foam $\left(41 \times 47 \times 8 \mathrm{~cm}\right.$; density $\left.=108 \mathrm{~kg} / \mathrm{m}^{3}\right)$ was used for the compliant surface condition. When standing on this type of foam, subjects were unable to feel the floor surface, thus, proprioceptive information from the feet and ankles was less reliable. Subjects practiced each postural task prior to the pre-fatigue trials. Subjects were asked to position their hands on their hips at the beginning of the trials and were allowed to use them to maintain their balance if necessary.

After completing a first bout of fatiguing exercise (IM or IK depending on session), subjects performed the post-fatigue postural trials in one condition (firm or compliant). Then, after completing the same fatiguing exercise a second time (second bout), subjects performed the post-fatigue postural trials in the other condition (compliant or firm). The fatiguing task was repeated between the two post-fatigue postural conditions in order to ensure the maintenance of an equivalent fatigue state for both conditions. The time elapsed between the end of the fatiguing exercise and the start of the first post-fatigue postural trial for each condition was $40 \mathrm{~s}$ on average. The order of the sessions (IM and IK session) and postural conditions (firm, compliant) were counterbalanced between subjects.

\section{Fatiguing exercises}

Both fatiguing exercises were completed using a Biodex system 3 dynamometer (Shirley, NY, USA) and a custom attachment allowing the production of force using plantarflexor muscles from both ankles simultaneously (Figure 5). During both sessions, subjects were first secured on the dynamometer in the initial position (Figure 5) where legs were horizontal, the trunk elevated to form a $25^{\circ}$ angle, and the angle between the legs and the feet fixed at $90^{\circ}$. Subjects performed 10 submaximal isokinetic contractions $\left(30^{\circ} \cdot \mathrm{s}^{-1}\right)$ and five submaximal isometric contractions of increasing intensities as a warm-up and to be familiarized with the dynamometer. Subsequently, for both IM and IK session, subjects performed three maximal isometric voluntary contractions $\left(\mathrm{MVC}_{\mathrm{IM}}\right)$ where the highest peak torque output was considered the subject's pre-fatigue $\mathrm{MVC}_{\mathrm{IM}}$. The same procedure was used to calculate the maximal voluntary isokinetic concentric contraction $\left(\mathrm{MVC}_{\mathrm{IK}}\right)$ before the IK fatiguing exercise (IK session only).

The fatiguing exercise for the IM session consisted of maintaining $50 \%$ of pre-fatigue $\mathrm{MVC}_{\mathrm{IM}}$ until exhaustion (i.e. subjects unable to maintain the force target for $3 \mathrm{~s}$ ). Whereas the fatiguing exercise for the IK session consisted of performing continuous maximal concentric IK 


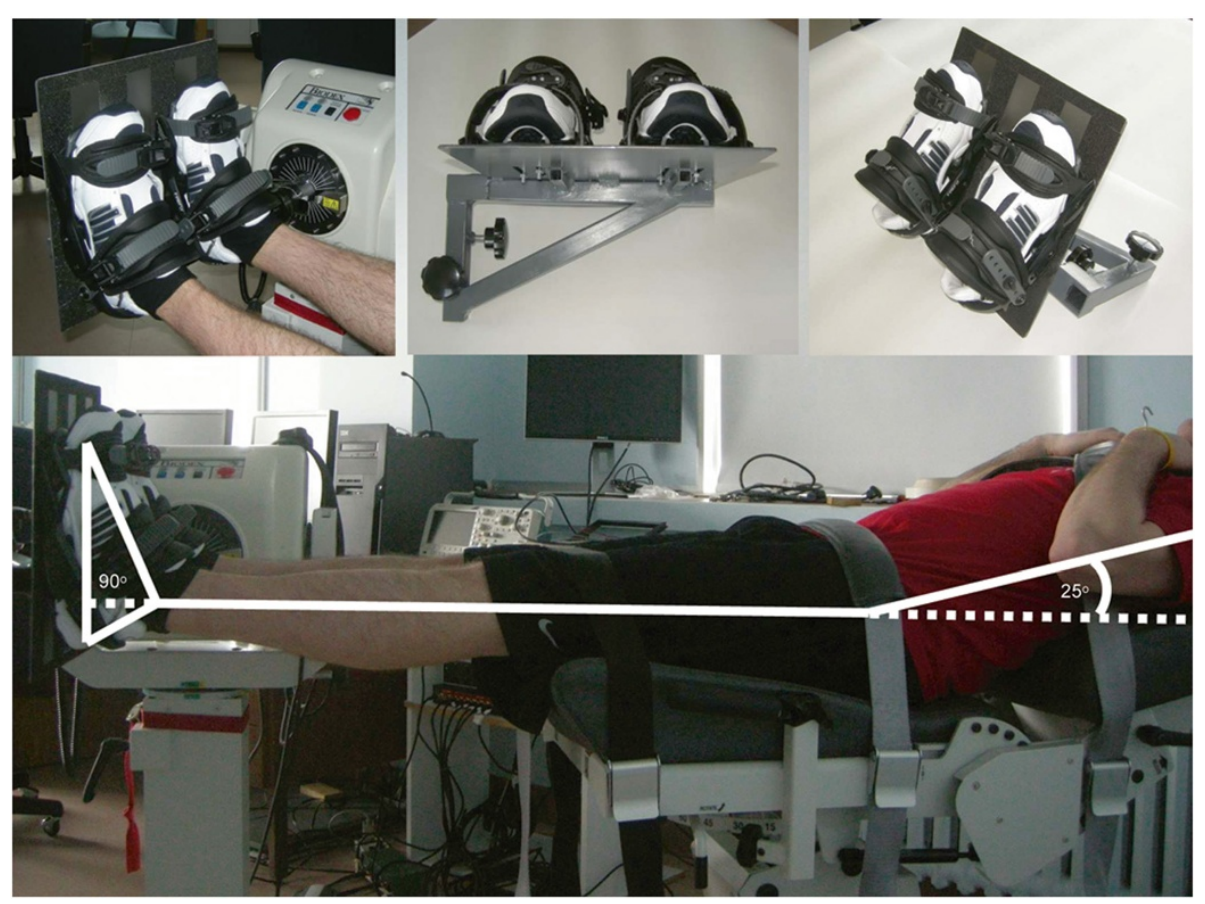

Figure 5 Experimental setup. Initial position for the MVCs and the fatiguing exercises. Also depicted is the custom attachment which allowed the simultaneous assessment of both ankles on the Biodex System 3.

contractions of the plantarflexors. Angular velocity was set at $30^{\circ} \cdot \mathrm{s}^{-1}$ with a total range of motion of $35^{\circ}$ (from $10^{\circ}$ dorsiflexion to $25^{\circ}$ plantarflexion, with $0^{\circ}=$ initial position). The isokinetic fatiguing exercise ended when the torque decreased below $50 \%$ of $\mathrm{MVC}_{\mathrm{IK}}$ for three consecutive contractions. To avoid any muscle fatigue of dorsiflexor muscles, after each plantarflexion contraction through the $35^{\circ}$ range of motion, an experimenter quickly pulled back the attachment to the starting position $\left(10^{\circ}\right.$ dorsiflexion) and subjects were instructed to relax during that time. After completion of the first and second bouts of fatiguing exercise for both IM and IK session, subjects were asked to perform a $\mathrm{MVC}_{\mathrm{IM}}$ (post-fatigue 1, post-fatigue 2) before transferring to the force platform to compare the level of fatigue after both types of exercise. Strong verbal encouragements and visual feedback of the torque output were provided to subjects during the MVC contractions and fatiguing exercises.

\section{Data acquisition}

The torque output from the dynamometer was sampled $(5000 \mathrm{~Hz})$ via a $1401 \mathrm{Plus}$ analog-to-digital board using Spike2 v.7 (CED, Cambridge, UK). CoP data were collected using an AMTI AccuGait force-platform (Watertown, MA, USA) at a $50 \mathrm{~Hz}$ sampling rate. The changes in $\mathrm{CoP}$ variations associated with fatigue were quantified with three postural variables using BioAnalysis 2.1 software (Watertown, MA, USA): the CoP excursion area $\left(\mathrm{cm}^{2}\right)$ represented by the $95 \%$ ellipse area [42], CoP variability $(\mathrm{cm})$ represented by the standard deviation of the $\mathrm{CoP}$, and mean CoP velocity $\left(\mathrm{cm} \cdot \mathrm{s}^{-1}\right)$. CoP variability and velocity variables were computed separately for the $\mathrm{ML}$ and AP directions. The mean of the three trials for each surface and fatigue conditions was calculated and used for statistical analyses.

\section{Data analysis}

A two-way analysis of variance (ANOVA) with repeated measures was used to assess the effects of fatigue (prefatigue, post-fatigue 1 and post-fatigue 2) and type of fatigue (IM and IK) on $\mathrm{MVC}_{\mathrm{IM}}$. A two-way ANOVA with repeated measures was used to assess the effects of fatigue (post-fatigue 1 and post-fatigue 2) and type of fatigue (IM and IK) on time to failure (the time elapsed to reach the fatigue state criterion of each fatiguing exercise). Separate three-way ANOVAs with repeated measures were used to assess the effects of fatigue (pre and post-fatigue), type of fatigue (IM and IK) and type of surface (firm and compliant) for each of the five postural variables (CoP excursion area, ML and $\mathrm{AP}$ CoP variability, ML and AP CoP velocity). For all statistical tests, the significance level was set at 0.05 . Post hoc analyses were completed when appropriate using Bonferroni adjustments. Normal distribution of the data was confirmed with Kolmogorov-Smirnov tests. 


\section{Competing interests}

None of the authors have any financial and non-financial competing interests related to this work

\section{Authors' contributions}

EJB conceptualized the study, carried out the data collection, statistical analyses and drafted the manuscript. AR and SB participated in the design of the study, the data collection, data analyses and the writing of the manuscript. YL participated in the analysis and interpretation of the data, and revised the manuscript. MB participated in the conception and design of the study and revised the manuscript. All authors read and approved the final manuscript.

\section{Acknowledgements}

This study was funded in part by a Canadian Institutes of Health Research Doctoral Award (Frederick Banting and Charles Best Canada Graduate Scholarships) and the "Fonds de la recherche en santé du Québec" Doctoral Award to Etienne J. Bisson and by a Canada Foundation for Innovation Leaders Opportunity Fund Award and a Natural Sciences and Engineering Research Council of Canada Discovery Grant Award to Martin Bilodeau.

\section{Author details}

${ }^{1}$ School of Human Kinetics, Faculty of Health Sciences, University of Ottawa Ottawa, Canada. ${ }^{2}$ Aging and Movement Research Laboratory, Bruyère Research Institute, Ottawa, Canada. ${ }^{3}$ School of Rehabilitation Sciences, Faculty of Health Sciences, University of Ottawa, Ottawa, Canada.

Received: 16 September 2011 Accepted: 15 May 2012

Published: 14 June 2012

\section{References}

1. Horak FB, MacPherson JM: Postural Orientation and Equilibrium. In Handbook of physiology, section 12: Exericse: Regulation and Integration of Multiple Systems. Edited by Rowell LB, Sheherd JT. NY: Oxford University Press; 1996:255-292

2. Bisson EJ, McEwen D, Lajoie Y, Bilodeau M: Effects of ankle and hip muscle fatigue on postural sway and attentional demands during unipedal stance. Gait Posture 2011, 33:83-87.

3. Boyas S, Remaud A, Bisson EJ, Cadieux S, Morel B, Bilodeau M: Impairment in postural control is greater when ankle plantarflexors and dorsiflexors are fatigued simultaneously than when fatigued separately. Gait Posture 2011, 34:254-259.

4. Bisson EJ, Chopra S, Azzi E, Morgan A, Bilodeau M: Acute effects of fatigue of the plantarflexor muscles on different postural tasks. Gait Posture 2010, 32:482-486.

5. Mello RG, Oliveira LF, Nadal J: Anticipation mechanism in body sway control and effect of muscle fatigue. J Electromyogr Kinesiol 2007, 17:739-746

6. Gribble PA, Hertel J: Effect of lower-extremity muscle fatigue on postural control. Arch Phys Med Rehabil 2004, 85:589-592.

7. Salavati M, Moghadam M, Ebrahimi I, Arab AM: Changes in postural stability with fatigue of lower extremity frontal and sagittal plane movers. Gait Posture 2007, 26:214-218.

8. Vuillerme $N$, Nougier $V$, Prieur JM: Can vision compensate for a lower limbs muscular fatigue for controlling posture in humans? Neurosci Lett 2001, 308:103-106

9. Vuillerme N, Danion F, Forestier N, Nougier V: Postural sway under muscle vibration and muscle fatigue in humans. Neurosci Lett 2002, 333:131-135.

10. Vuillerme N, Forestier N, Nougier V: Attentional demands and postural sway: the effect of the calf muscles fatigue. Med Sci Sports Exerc 2002, 34:1907-1912

11. Vuillerme N, Nougier V: Effect of light finger touch on postural sway after lower-limb muscular fatigue. Arch Phys Med Rehabil 2003, 84:1560-1563.

12. Loram ID, Maganaris CN, Lakie M: Paradoxical muscle movement in human standing. J Physio/ 2004, 556:683-689.

13. Corbeil P, Blouin JS, Begin F, Nougier V, Teasdale N: Perturbation of the postural control system induced by muscular fatigue. Gait Posture 2003, 18:92-100.
14. Gandevia SC: Spinal and supraspinal factors in human muscle fatigue. Physiol Rev 2001, 81:1725-1789.

15. Boyas S, Guével A: Neuromuscular fatigue in healthy muscle: Underlying factors and adaptation mechanisms. Ann Phys Rehabil Med 2011 54:88-108.

16. Forestier N, Teasdale N, Nougier V: Alteration of the position sense at the ankle induced by muscular fatigue in humans. Med Sci Sports Exerc 2002 34:117-122.

17. Hiemstra LA, Lo IK, Fowler PJ: Effect of fatigue on knee proprioception implications for dynamic stabilization. J Orthop Sports Phys Ther 2001, 31:598-605.

18. Enoka RM, Duchateau J: Muscle fatigue: what, why and how it influences muscle function. J Physiol 2008, 586:11-23.

19. Babault N, Desbrosses K, Fabre MS, Michaut A, Pousson M: Neuromuscular fatigue development during maximal concentric and isometric knee extensions. J Appl Physiol 2006, 100:780-785

20. Lysholm M, Ledin T, Odkvist LM, Good L: Postural control-a comparison between patients with chronic anterior cruciate ligament insufficiency and healthy individuals. Scand J Med Sci Sports 1998, 8:432-438.

21. Guskiewicz KM: Postural stability assessment following concussion: one piece of the puzzle. Clin J Sport Med 2001, 11:182-189.

22. Horak FB, Shupert CL, Mirka A: Components of postural dyscontrol in the elderly: a review. Neurobiol Aging 1989, 10:727-738.

23. Shumway-Cook A, Woollacott M: Attentional demands and postural control: the effect of sensory context. J Gerontol A Biol Sci Med Sci 2000, 55:M10-M16.

24. Tremblay F, Mireault AC, Dessureault L, Manning H, Sveistrup H: Postural stabilization from fingertip contact: I. Variations in sway attenuation, perceived stability and contact forces with aging. Exp Brain Res 2004, 157:275-285.

25. Woollacott MH, Shumway-Cook A, Nashner LM: Aging and posture control: changes in sensory organization and muscular coordination. Int J Aging Hum Dev 1986, 23:97-114.

26. Lord SR, Tiedemann A, Chapman K, Munro B, Murray SM, Sherrington C: The effect of an individualized fall prevention program on fall risk and falls in older people: a randomized, controlled trial. J Am Geriatr Soc 2005, 53:1296-1304

27. Paillard T: Effects of general and local fatigue on postural control. A review. Neurosci Biobehav Rev 2011, 36:162-176.

28. Reimer RC III, Wikstrom EA: Functional fatigue of the hip and ankle musculature cause similar alterations in single leg stance postural control. J Sci Med Sport 2010, 13:161-166.

29. Surenkok O, Kin Isler A, Aytar A, Gultkin Z, Akman M: Effect of knee muscle fatigue and lactic acid accumulation on balance in healthy subjects. Isokinet Exerc Sci 2006, 14:301-306.

30. Dickin DC, Doan JB: Postural stability in altered and unaltered sensory environments following fatiguing exercise of lower extremity joints. Scand J Med Sci Sports 2008, 18:765-772.

31. Frey Law LA, Avin KG: Endurance time is joint-specific: a modelling and meta-analysis investigation. Ergonomics 2010, 53:109-129.

32. Okada M: An electromyographic estimation of the relative muscular load in different human postures. J Hum Ergol (Tokyo) 1973, 1:75-93.

33. Vuillerme N: Differential integration of kinaesthetic signals to postural control. Exp Brain Res 2006, 174:763-768.

34. Maurer C, Mergner T, Peterka RJ: Multisensory control of human upright stance. Exp Brain Res 2006, 171:231-250.

35. Vuillerme N, Pinsault N: Re-weighting of somatosensory inputs from the foot and the ankle for controlling posture during quiet standing following trunk extensor muscles fatigue. Exp Brain Res 2007, 183:323-327.

36. Peterka RJ: Sensorimotor integration in human postural control. J Neurophysiol 2002, 88:1097-1118.

37. Ruhe A, Fejer R, Walker B: The test-retest reliability of centre of pressure measures in bipedal static task conditions-a systematic review of the literature. Gait Posture 2010, 32:436-445

38. Allen TJ, Leung M, Proske U: The effect of fatigue from exercise on human limb position sense. J Physio/ 2010, 588:1369-1377.

39. Vuillerme $\mathrm{N}$, Boisgontier M: Muscle fatigue degrades force sense at the ankle joint. Gait Posture 2008, 28:521-524. 
40. Winter DA: Unified theory regarding $\mathrm{A} / \mathrm{P}$ and $\mathrm{M} / \mathrm{L}$ balance in quiet stance. J Neurophysiol 1996, 75:2334-2343.

41. Day BL, Steiger MJ, Thompson PD, Marsden CD: Effect of vision and stance width on human body motion when standing: implications for afferent control of lateral sway. J Physiol 1993, 469:479-499.

42. Advanced Mechanical Technology Inc: BioAnalysis Version 2.2. MA: Watertown; 2004.

doi:10.1186/1743-0003-9-39

Cite this article as: Bisson et al:: Effects of fatiquing isometric and isokinetic ankle exercises on postural control while standing on firm and compliant surfaces. Journal of NeuroEngineering and Rehabilitation 2012 9:39.

\section{Submit your next manuscript to BioMed Central and take full advantage of:}

- Convenient online submission

- Thorough peer review

- No space constraints or color figure charges

- Immediate publication on acceptance

- Inclusion in PubMed, CAS, Scopus and Google Scholar

- Research which is freely available for redistribution 